(Aus der Universitäts-Augenklinik des Hofrats Prof. E. Fuchs in Wien.)

\title{
Über die Abhebung der Pars ciliaris retinae und das Abreissen der Retina an der Ora serrata.
}

\author{
Von \\ Dr. Rudolf Bergmeister, \\ Klinischer Assistent. \\ Mit Taf, XXI, Fig. 1-6.
}

Es ist eine kaum bekannte Tatsache, dass es Abhebungen der Pars ciliaris retinae gibt. Im allgemeinen gilt es ja als Regel, dass auch totale Netzhautablösungen nur bis an die Ora serrata reichen. Als Ausnahme wird ein Fall ron Velhagen (Arch. f. Ophth. 1900) citiert. Velhagen beschreibt eine Abhebung der Cylinderzellen der Pars ciliaris retinae durch Exsudat infolge von traumatischer Iridocyclitis. Ein in der Nähe der Ora serrata fixierter Bindegewebsstrang hatte gleichzeitig die Cylinderzellenlage von ihrer Unterlage abgerissen.

Ausserdem ist in der bekannten Monographie von Nordenson (1887) erwähnt, dass an einer allgemeinen Netzhautablösung mehr oder weniger die Pars ciliaris beteiligt sein kann ${ }^{1}$ ). Dass diese Abhebungen der Pars ciliaris doch vielleicht häufiger vorkommen und grösseres Interesse beanspruchen, soll in folgendem gezeigt werden.

Gleich hier sei bemerkt, dass unter Pars ciliaris retinae nur die unpigmentierte Lage der Cylinderzellen verstanden wird.

Abhebungen beider Blätter der unpigmentierten und pigmentierten Lage scheint zu den grössten Seltenheiten zu gehören; dies konnte nur in zwei Präparaten gefunden werden. Beide betrafen Chorioidealsarkome mit totaler Netzhautablösung. Im hintersten Abschnitt des Ciliarkörpers (glatte Zone Salzmanns) war das Pigmentepithel von der Lamina vitrea chorioideae abgelöst, während die Cylinderzellen in unverändertem Zusammenhang mit der Unterlage waren. Die subretinale Flüssigkeit war zwischen Pigmentepithel und Lamina vitrea getreten (Taf. XXI, Fig. 1).

Die Abhebungen der eigentlichen Pars ciliaris retinae (unpigmen-

1) v. Hippel (v. Graefe's Arch. Bd. LXVIII) sieht in diesen Fällen einen Beweis für die Zugwirkung des Glaskörpers bei Ablatio retinae. Vgl, auch Leber (Heideiberger Vers. 1908). 
tierte oder Cylinderzellenlage) können entweder isoliert (ohne Beteiligung der Pars optica) oder in continuo mit einer allgemeinen Netzhautablösung vorkommen.

Ätiologisch kann als Einteilungsprinzip folgendes angenommen werden. Die Abdrängung der Cylinderzellenlage kann erfolgen:

1. Durch einen serösen Erguss (primäre Netzhautabhebung, Tumor).

2. Durch Exsudation unter dem Epithel infolge von akuter oder chronischer Entzündung des Ciliarkörpers oder der angrenzenden Teile der Chorioidea.

3. Durch Blutung aus den Aderhautgefässen (expulsive Hämorrhagie).

4. Ausfliessen des Glaskörpers (wie etwa nach perforierenden Verletzungen, Skleralrupturen), ferner schrumpfende Schwarten der Ciliargegend können die Cylinderzellen der Pars ciliaris zur Abhebung bringen, wobei als ursächliches Moment eine Zugwirkung angenommen werden muss.

Die isolierten Abhebungen der Pars plana können unter Umständen als Cysten imponieren. Zufällig fanden sich bei der Durcharbeitung dieses Themas zwei sehr präguante Fälle, die hier mitgeteilt werden sollen.

Solche cystenartige Abhebungen der Pars ciliaris retinae fanden sich in dem Auge eines 57jährigen Patienten, welches wegen eines Aderhautsarkoms enueleiert wurde, und in dem Auge eines 47jährigen Patienten, welches infolge einer (durch Kuhbornstoss entstandenen) Skleralruptur am Tage nach der Verletzung zur Enucleation kam. Die Cyste beginnt unmittelbar an der Ora serrata, welch letztere in festem Zusammenhang mit dem Pigmentepithel steht. Die Cylinderzellen der Pars ciliaris retinae sind durch eine eiweissreiche Flüssigkeit gegen das Augeninnere rorgebaucht, in dem Fall von Aderhautsarkom prall gespannt und flachgedrickt, so dass ihre Form kaum zu erkennen ist. Das Pigmentepithel ist in den Ciliarkörper hineingedrängt (Taf. XXI, Fig. 2).

In dem Falle von Skleralruptur hatten die Cylindèrzellen ihre Form noch unverändert erhalten, das Pigmentepithel wies die gleiche Ausbauchung gegen die Chorioidea zu auf. Der Oysteninhalt bestand aus geronnener Flüssigkeit mit einigen desquamierten Epithelzellen. Ähnliche Befunde wurden zuerst von Kuhnt beschrieben, der sie als Altersveränderungen dentete (Heidelberger Versammlung 1881). Er glaubte nämlich, dass die gleichzeitig vorhandenen atrophischen 
Über d. Abhebung d. Pars ciliaris retinae u. d. Abreissen d. Retina usw. 553

Prozesse in den Gefässbezirken des Corpus ciliare die Ursache der Transsudation sind. Dies bewirkt dann eine cystische Abhebung der Pars ciliaris retinae. In dem ersterwähnten Falle wäre diese angegebene Ursache einer Cirkulationsstörung sehr plausibel, nachdem ein Ausläufer des Aderbautsarkoms in Form des infiltrativen Wachstums einen Teil des Ciliarkörpers ergriffen hatte. Eine ganz andere Erklärung muss man in dem Falle von Skleralruptur heranziehen. Zunächst ist hier die Tatsache hervorzuheben, dass das Abfliessen einer grösseren Menge von Glaskörperflüssigkeit eine Abhebung der Pars ciliaris zur Folge hat. Noch bemerkenswerter ist das Fehlen einer gleichzeitigen Abhebung der Pars optica. Die isolierte Abhebung der Pars ciliaris plana erinnert an die Befunde, die Greeff nach Punktion der Vorderkammer des Kaninchenauges feststellte. Es waren dies blasige Abhebungen des Epithelzellenüberzuges der Pars plicata des Ciliarkörpers. Der Blaseninhalt bestand aus geronnener Flüssigkeit (dem eiweissreichen Kammerwasser entsprechend). Die Basis der Blase bildete das bindegewebige Stroma des Ciliarkörpers selbsto1). Ähnlich wie die Punktion der Vorderkammer hatte die Ruptur der Sklera rapiden Flüssigkeitsabfluss aus dem Augeninnern zur Folge gehabt, der Veranlassung zum Austritt von Flüssigkeit in und unter die Epithelschicht der Pars plana des Ciliarkörpers gab.

In die grosse Gruppe der in Kontinuität mit einer allgemeinen Netzhautablösung vorkommenden Abhebungen der Cylinderzellenlage der Pars ciliaris gehören zunächst Fälle von serösen Netzhautabhebungen (primär oder durch einen Tumor bedingt). Wie schon oben erwähnt gilt es ja für die primären, serösen Netzhhautabhebungen als Regel, dass die Ablösung ihre Grenze an der Ora serrata findet. Trotzdem kam ein hochgradig myopisches Auge (von $13 \mathrm{D}$. mit Achsenverlängerung) zur Beobachtung, indem die totale Netzhautablösung sich auch auf die Pars plana erstreckte 2). Das Auge selbst kam wegen Iridocyclitis (Occlusio pupillae) und Sekundärglaukom zur Enucleation. Die Netzhaut befand sich bereits in dem Stadium einer cystischen Degeneration, die Limitans interna war durch Flüssigkeit abgelöst und als Strang hinter der Linse gelegen. Am Übergang der Chorioidea in den Ciliarkörper fand sich eine schwartige Auflagerung, die als ringtörmiger Wulst der ganzen Ora serrata entlang läuft, wie man es ja in Augen mit alter

1) Auch Kuhnt erwähnt, dass mitunter beide Blätter der Pars ciliaris plana abgehoben sein können.

2) Vgl. Leber (Heidelberger Vers. 1908), 
Netzhautablösung des öfteren findet. Trotz dieser Schwarte sind die Cylinderzellen von derselben durch einen serös hämorrhagischen Erguss getrennt und treten erst am vorderen Rand mit der Schwarte in Verbindung. Es ist also sehr wahrscheinlich, dass die Cylinderzellen schon in dem ersten Stadium der Netzhautabhebung mit abgelöst wurden, lange bevor noch die Schwarte gebildet wurde (Taf. XXI, Fig. 3).

Gleichzeitig konnte ein Einriss in den rückwärtigen Teil der Cylinderzellenlage konstatiert werden, der zum Teil von dem Bindegewebe der chorioiditischen Auflagerung verschlossen ist $\left.{ }^{1}\right)$. Von diesem Riss nehmen eigenartige Proliferationsvorgänge des Ciliarepithels ihren Ausgang. Diese führten zu einer epithelartigen Bedeckung der chorioiditischen Schwarte mit ganz flachen Zellen (Taf. XXI, Fig. 3 u. 6),

In ungefähr 12 Fällen von Aderhautsarkom mit Netzhautablösung fand sich ein mehr oder weniger grosser Anteil der Cylinderzellenlage mit abgelöst.

Gewöhnlich findet sich die Ablösung auf derselben Seite wie der Tumor und ist zwischen Cylinderzellen und Pigmentepithel subretinale Flüssigkeit mit Tumorzellen gemengt nachzuweisen. Zumeist bestand gleichzeitig Sekundärglaukom. Selbst bei einem ganz ldeinen Tumor im hinteren Bulbusabschnitt kam es neben ausgedehnter Netzhautablösung anch zur Abhebung der Cylinderzellen der Pars ciliaris, wobei die Oylinderzellenlage weit vorne (ausnahmsweise) fast am Beginn der Pars plicata abgerissen war (Taf. XXI, Fig. 4).

Es ist geradezu auffallend, dass in einer nicht geringen Anzahl von Netzhautablösungen bei Tumoren auch gleichzeitig ein Abreissen der Netzhaut an der Ora serrata zu konstatieren ist. Fast immer ist gleichzeitig die Parş ciliaris retinae abgehoben. Es würde dies für eine Entstehung des Einrisses erst in einem späten Stadium sprechen, da bei frühzeitigem Abreissen der Retina von der Ora serrata es nicht zur Ablösung der Pars ciliaris retinae kommen könnte.

Vorbereitet wird dieses Abreissen der Netzhaut ganz entschieden durch das Vorhandensein der Blessigschen Hohlräume. Die Abreissung findet sich zumoist über dem Tumor.

Bestehen im Tumor selbst oder auch im Ciliarkörper ausgedehntere Gewebsnekrosen, so können abgehobene Cylinderzellen der Pars eiliaris ungemein rasch nekrotisch werden, während die iubrige abgehobene Netzhatut noch gute Zellfärbung aufweist. Diese Bilder imponieren unter Umständen als Abreissungen der Retina an der Ora serrata.

j) Auch dieser Riss Kann erst später nach Ablusung der Cylinderzellenage aufgetreten sein! 
Über d. Abhebung d. Pars ciliaris retinae u. d. Abreissen d. Retina usw. ว̌5う

Abdrängung des Cylinderepithels bei akuter und chronischer Entzündung ist tatsächlich zu beobachten. Es ist hervorzuheben, dass in diesen Fällen auch die Pars plicata entweder allein oder mit der Pars plana abgehoben wird.

In einem Auge, das wegen einer postoperativen Iridocyclitis (Excision einer Iriscyste) enucleiert werden musste, war die Netzhaut in toto durch ein seröses Exsudat abgehoben. Gleichzeitig war eine intensive Entzündung der Retina (Endophthalmitis nach Fuchs) vorhanden. Die Abhebung erstreckte sich in continuo auch auf die Cylinderzellenlage nicht nur der Pars plana, sondern auch der Pars plicata. Der seröse (subrenitale) Erguss war besonders im Bereich des Ciliarkörpers von zahlreichen Eiterkörperchen durchsetzt.

In einem Falle von Ringabscess nach einfacher Linearextraktion kam es zu cystischen Abhebungen der Cylinderzellen der Pars plicata infolge von Exsudation und Ansammlung von Leukocyten, während die Pars plana nicht abgehoben wurde.

Ähnliche Abhebungen der Pars plicata können nicht nur durch akute, eitrige Entzündungen, sondern auch durch chronische Entzündungen hervorgebracht werden.

In einem Falle von Tuberkulose der Iris und des Ciliarkörpers hatte das tuberkulöse Granulationsgewebe das Pigmentepithel durchbrochen und die Cylinderzellen abgehoben. Letztere zeigen keinerlei Zeichen beginnender Nekrose, obwohl das tuberkulöse Granulationsgewebe im Bereich der Iris beide Blätter des Epithels zum Verschwinden gebracht hat (Taf. XXI, Fig. 5).

Blutung aus den A derhautgefäs sen in Fällen von Skleralruptur oder nach Glaukomiridektomie können die Pars optica und im Zusammenhang damit einen mehr oder weniger grossen Teil der Pars plana abheben.

Besonderes Interesse bieten die Abhebungen, die durch Ausfliessen einer mehr oder weniger grossen Glaskörpermenge zu stande kommen, so insbesondere nach frischer Skleralruptur (vgl. obigen Fall mit cystischer Abhebung der Pars plana).

Es handelt sich zumeist um eine Fortsetzung der allgemeinen Netzhautabhebung, die bald mit, bald ohne Kontinuitätsunterbrechung an der Ora serrata die Cylinderzellenlage mit abgelöst hat und zwar teilweise nicht nur in der glatten Zone, sondern auch in der Gross- und Kleinmaschenzone (siehe Salzmann, die Zonula ciliaris, 1900), während die Cylinderzellen der Pars plicata auf ihrer Unterlage haften bleiben ${ }^{1}$ ).

1) Es fanden sich nur einzelne kleinere cystische Abhebungen der Cylinderzellen rom Typus der vorhin geschilderten. 
Eine Zugwirkung der Zonulafasem auf die Cylinderzellen konnte nicht mit Sicherheit angenommen werden, wenn auch die Linse vollständig aus dem Bulbus herausgeschleudert war. Auch die auf den abgehobenen Cylinderzellen liegende, innere Glashaut (Salzmanns), in die ja Zonulafasern einstrahlen, wies keine Loslösung yon ihrer Unterlage auf. Hingegen konnten in den Tälern und Leisten der Pars plicata deutlich abgerissene Zonulafasern gefunden werden, die in festem Zusammenhang mit den Cylinderzellen geblieben waren. Es steht dies so ziemlich im Einklang mit den Untemehmungen Salzmanns. Die Zonulafasermasse ist am vorderen Rande des Orbiculus ciliaris am meisten befestigt und zwar an der inneren Glashaut, die gerade hier ein System von Leisten aufweist. Hier lassen sich die Zonulafibrillen mur bis zur inneren Giashaut und nicht darüber hinaus verfolgen. Im rïckwärtigen Abschnits (Bereich der Grossmaschenzone des Pigmentepithels und der hohen Cylinderzellen) strahlen die Zonulafasern in die sogenannte Ciliarzone der Hyaloidea und in die vordere Grenzschicht des Glaskörpers ein. Präpariert man den Glaskörper, so gehen die hinteren Zonulaurspriinge mit, während sie vorne abreissen.

So konnte die Pars plicata nicht zur Ablösung gebracht werden bei der Ruptur eines bereits staphylomatösen Auges (durch einen Schneeballwurf). Trotzdem waren Teile der Cylinderzellenlage der Pars plana herausgerissen und in die Skleralwunde zu liegen gekommen, welch letztere ventilartig von einem Bindehautlappen bedeckt war.

Sonderbar war auch der Befund in einem Präparat, das von einem an Keratomalacie erkrankten Kinderange starnmte. Es bestand seichte totale Ablösung der Netzhant, die sich auch auf die Pars plana erstreckte. Da die Linse infolge der Perforation in dem Auge fehlte, so muss angenommen werden, dass der Zug des ausfliessenden Glaskörpers die Abhebung bewirkte. Es ist dies um so wahrscheinlicher, als im Auge des Neugeborenen sicherlich der Glaskörper in viel festerem Zusammenhang mit der Netzhaut ist, als im Auge des Erwachsenen. Ist ja doch nach den heutigen Anschauungen das Auswachsen der Glaskörperfibrillen aus den versohiedensten Teilen der Netzhaut im Fötalleben sichergestellt; ferner dass das Auswachsen von Fibrillen aus der Pars caeca am längsten anzudauern scheint, etwa bis das Auge seine definitive Grösse erreicht hat. (S. Wolfrum LXV. Bd. v. Graefe's Arch. Szily, Kölliker Zeitschr. f, wiss. Zool. 1.904.) 
Über d. Abhebung d. Pars ciliaris retinae u. d. Abreissen d. Retina usw. 557

Eine grosse Anzahl von Abhebungen der Pars ciliaris retinae mit und ohne Abreissung ist durch $Z$ ug einer schrumpfenden Schwarte verursacht, am häufigsten nach traumatischer Iridocyclitis.

So fand sich in einem Auge nach Cornealverletzung an Stelle der Linse eine mächtige Schwarte, die auf der einen Seite mit der vernarbten $W$ unde verwachsen war. Von der Linse ist nur mehr die gefaltete Linsenkapsel erhalten, von der Iris ist nichts mehr zu sehen. Der der Narbe gegenüberliegende Teil der Schwarte ist in der Gegend der Ora serrata mit der Retina verwachsen. Diese Verwachsung hatte eine vollständige. Ablösung der Pars plana zu stande gebracht. Mit Ausnahme einiger Falten in der Nähe der Ora serrata erscheint die abgelöste Partie der Cylinderzellen geradegestreckt. Knapp am Übergang in die Pars plicata ist ein Riss vorhanden. Höchstens kann man von einem Bindeglied, bestehend aus wenigen Fasern, sprechen. Der Riss kann zur Erklärung einer merkwürdigen Veränderung des blossgelegten Pigmentepithels der Pars plana dienen. Das Pigmentepithel liegt nicht frei da unter der subretinalen Flüssigkeit, sondern ist von einer Reihe flacher pigmentloser Zellen überzogen, die etwa in der Gegend der Ora serrata ein plötzliches Ende finden. Offenbar ist es von dem Rissrand aus zu einer regeneratorischen Proliferation des Cylinderepithels gekommen (Taf. XXI, Fig. 6).

In einem Fall von Iridocyclitis plastica nach einer Ruptur, der vier Jahre nach der Verletzung zur Enucleation kam, waren auf einer Seite die Cylinderzellen der Pars ciliaris isoliert abgehoben und dadurch eine Art Cystenraum entstanden, da die Ora serrata durch einen entzündlichen Prozess mit der Unterlage fest verbunden war. Selbst in dem Hohlraum dieser Cyste fanden sich proliferierende Epithelsprossen, die zum Teil Brücken zwischen Pigmentepithel und Cylinderzellen bildeten.

Ein anderes Mal tritt die regenerative Proliferationstätigkeit der Cylinderepithelzellen noch mehr in den Vordergrund. Die Proliferationstätigkeit kann zu ziemlich ausgedehnter Cystenbildung der Pars plana Anlass geben, und sehr oft sind die neugebildeten Zellen nicht von den ursprünglichen Cylinderzellen zu unterscheiden. Höchstens dass an einer bestimmten Stelle der Cystenwand ein konzentrisch geschichtetes Gebilde (wie eine Knospe aussehend) liegt mit Zellkernen, welches in seinem Ansehen einige Ähnlichkeit besitzt mit den senilen Excrescenzen des Ciliarepithels, wie sie Kuhnt beschreibt. Ist also die pars ciliaris retinae durch irgend eine Ursache abgehoben, so kann es, insbesondere dann, wenn eine Kontinuitäts- 
unterbrechung an der Ora serrata stattgefunden hat, zur Neubildumg von unpigmentierten Epithelzellen kommen. Der eine Typus ist repräsentiert durch die Bildung von Cysten und ist der seltenere. Der zweite ist die Bildung endothelartiger Zellen von der Rissstelle aus, die entweder direkt auf dem Pigmentepithel liegen, oder selbst eine chorioiditische Schwarte überziehen können (Taf. XXI, Fig. 4, 6).

Besonders aufmerksam zu machen ist darauf, dass schrumpfende Schwarten, die der Ora serrata anhaften, diese za einem Strang ausziehen können, der dann eventuell bis zur Mittellinie des Bulbus vorspringt. Die Oylinderzellen der Pars ciliaris sind infolge dieses Zuges mitunter gar nicht oder im Bereich der Pars plana abgehoben. Dieses Ausziehen der Ora serrata in Form einer Falte ist bereits Treacher Collins' bekannt gewesen (siehe Ophthalm. Hosp. Rep. XII. 4). Doch glaubt er in manchen Fällen den Zug der Zonulafasern als Ursache ansehen zu müssen.

Anhangsweise möchte ich noch einige interessante Fälle von $\mathrm{Ab}$ reissung der Retina schildern. So fand ich in einem atrophischen Bulbus nach Cornealverletzung an der Hinterfläche der Linse ein relatir kleines, in Schrumpiung begriffenes Exsudat, welches einerseits diesen Teil der Linsenkapsel zur Faltung gebracht, anderseits die Ora serrata bis hinter den Linsenäquator ausgezogen und die Pars plana zur Ablösung und Abreissung gebracht hatte. Der Riss war nicht in der Ora serrata selbst, sondem etwas weiter vorn innerhalb der Cylinderzellen. Es hatte eine Verheilung des Risses stattgefunden, da auf der entblössten Pars plana eine Zellproliferation stattgefunden hatte. Der abgerissene Rand der Netzhaut konnte hierdurch an einer viel weiter vorn liegenden Stelle wieder zur Anwachsung kommen. Entsprechend der Ora serrata fand sich im Pigmentepithel eine Lücke, durch welche ein Strom ron Eiterkörperchen aus der Aderhaut auswanderte und in den subretinalen Raum gelangte. Unter den abgehobenen Cylinderzellen der Pars plana fand sich ein Blutergusș. Das Präparat ist bemerkenswert wegen des akuten Entzündungszustandes und der gleichzeitig beginneuden Organisierung des Exsudats, Dabei hatte sich schon frithzeitig eine Proliferationstätig. keit der Cylinderzellen in der Gegend des Rissrandes entwickelt.

Ein anderes Mal konnte an dem etwas abgelösten vorderen Rissrande (der Pars ciliaris) eine keulenförmige Verdickung konstatiert werden, die, obwohl aus proliferierenden Cylinderzellen hervorgehend, bereits eine mehr oder weniger faserige Struktur zeigte. Der andere Risspand (der Pars optica) wax an einer Schwarte fixiert. In einem 
C̈ber d. Abhebung d. Pars clliaris retinae u. d. Abreissen d. Retina usw. 559 bereits staphylomatösen Auge fand sich ein stark gedehntes, faseriges Gewebe zwischen Pars optica und Cylinderzellen, so dass die Verheilung eines bestandenen Risses wahrscheinlich ist. Die Pars plana war durch frisches, eitriges Exsudat abgehoben, das aus der Chorioidea stammend die Pigmentepithelzellen siebförmig durchbrochen hatte.

In einem andern Falle hatte eine bindegewebige Masse hinter der Linse die Netzhaut gerade an der Ora serrata abgerissen. Die Abreissung war in diesem Fall cirkulär, die Cylinderzellenlage nicht abgehoben. Es wäre dies eine reine totale Dialysis retinae an der Ora serrata, die eine Abhebung der Cylinderzellen von vornherein ausschliesst.

Wenn somit, wie leicht erklärlich; Fälle von Ablatio der Pars ciliaris zur klinischen Diagnose niemals kommen, so scheint dies weniger für die Dialysis retinae zu gelten. 'So beschreibt Hesse (Zeitschr. f. Augenheilk. 1907) einen Fall von Netzhautablösung mit Abreissung in einem myopischen Ange. Die Netzhaut war sehr weit vorn abgerissen. und die rückwärtige Seite der Retina blossgelegt. Römer (Klin. Monatsbl. Bd. XXXIX. S. 106) beschreibt eime traumatische, totale Netzhautabhebung mit völliger Abreissung von der Peripherie, wobei das vordere Ende der Netzhaut samt der verlagerten Iris in einer in der Sklera gelegenen Perforationsnarbe fixiert war. Mit dem Augenspiegel konnte man auf die äussere Seite der Netzhaut blicken und dabei die Chorioidea mit dem Pigmentepithel frei daliegen sehen.

Einen höchst interessanten Fall von Netzhautabreissung an der Ora serrata schildert Fuchs (Klin. Monatsbl. Bd. XV. 1877). Es handelte sich um ein Auge mit einer geschrumpften, traumatischen Katarakt, in welchem zunächst eine optische Iridektomie nach innen unten angelegt wurde. Nach einer Discission per scleram war folgender Befund zu erheben: In dem Raum zwischen dem Rand der nach aussen gerückten Katarakt und den Ciliarfortsätzen konnte man bei seitlicher Beleuchtung auf der Oberfläche der seidenähnlich glänzenden Hyaloidea eine Anzahl sehr zarter, trüber Streifen erkennen. Man konnte deutlich dieselben an einer gefalteten Membran ihren Ansatz nehmen sehen, die sich als die abgerissene Ora serrata der nach rückwärts zu abgelösten Netzhaut erwies.

Der Fall ist deshalb bemerkenswert, weil bei der Reklination der Katarakt die Retina eher an der Ora serrata abriss, als dass die Zonulafasern gerissen wären. 
Dies setzt grössere Resistenz der Zonulafasern überhaupt sowie festere Insertion derselben am Linsenrand einerseits, an der Retina anderseits voraus, wozu noch eine Prädisposition zur Abhebung anzunehmen ist, da ja am andern Auge ebenfalls Netzhautabhebung entstanden war.

Als Resultat der vorliegenden Untersuchungen möchte ich kurz folgendes resümieren. Die Abhebungen der Cylinderzellenlage der Pars plana ist nicht so selten, wie bis jetzt angenommen wurde. Fs besteht ferner sicherlich infolge der Struktur der Netzhaut an der Ora serrata eine Prädisposition zum Ein- oder Abreissen. (Siehe die prallgespannten Abhebungen bei Tumoren - Vorbereitung des Abreissens durch Blessigsche Hohlräume.) Immerhin lann auch ausnahmsweise eine Kontinuitätsunterbrechung auch weiter vorn, innerhalb der Cylinderzellenlage vorkommen. Abhebung der Cylinderzellen und Einrisse in der Ora serrata oder der Pars ciliaris können auch kombiniert vorkommen.

Weinem verebrten Chef, Herrn Hofrat Fuchs, bin ich für das entgegengebrachte Interesse und für die Förderung dieser Arbeit zu grossem Dank verpflichtet.

Erklärung der Abbildungen auf Taf. XXI, Fig, 1-6.

Fig. 1. Ablösung beider Zellagen der glatten Zone des Ciliarkörpers. (Totale Netzhautabhebung bei Chorioidealsarkom.)

c. Ciliarkörper. $7 . v$. Lamina vitrea. o.s. Ora serrata nur noch mit wenigen Fasern mit den Cylinderzellen zusammenhängend.

Fig. 2. Isolierte Abhebung der Cylinderzellen der Pars plana nach frischer Skleralruptur.

Fig. 3. Ablösung der Pars ciliaris in einem hochgradig myopischen Auge mit Riss $\left(\boldsymbol{R}_{\text {. }}\right)$ innerhalb der Cylinderzellenlage.

ch. Chorioidea. c. Ciliarkörper. o. s. Ora serrata, $n$. Z. Neugebildete Zellen auf der Oberfüche der schwartigen Autlagerung.

Fig. 4. Totale Abhebung der Pars ciliaris plana (Ablatio retinae bei einem kleinen Chorioidealsarkom $\rangle_{*}$ Einriss weit vorn am Ubergang in die Pars plicata.

o. s. Ora serrata s. $\mathbb{F}$. Subretinale Flüssigkeit.

Fig. 5. Abhebung der Cylinderzellen bei Tuberkulose der Iris- und des Ciliarkörpers.

p. c. Cylinderzellen der Pars ciliaris.

Fig. 6. Ausziehung der Ora serrata (o.s.) und Abhebung der Pars ciliaris durch Zug einer cyclitischen Sehwarte $\left(S_{.}\right)$.

p. pl. Pars plana. n. $Z$. Neugebildete Zellen von der Rissstelle an der Pars plicata ausgehend. 
J
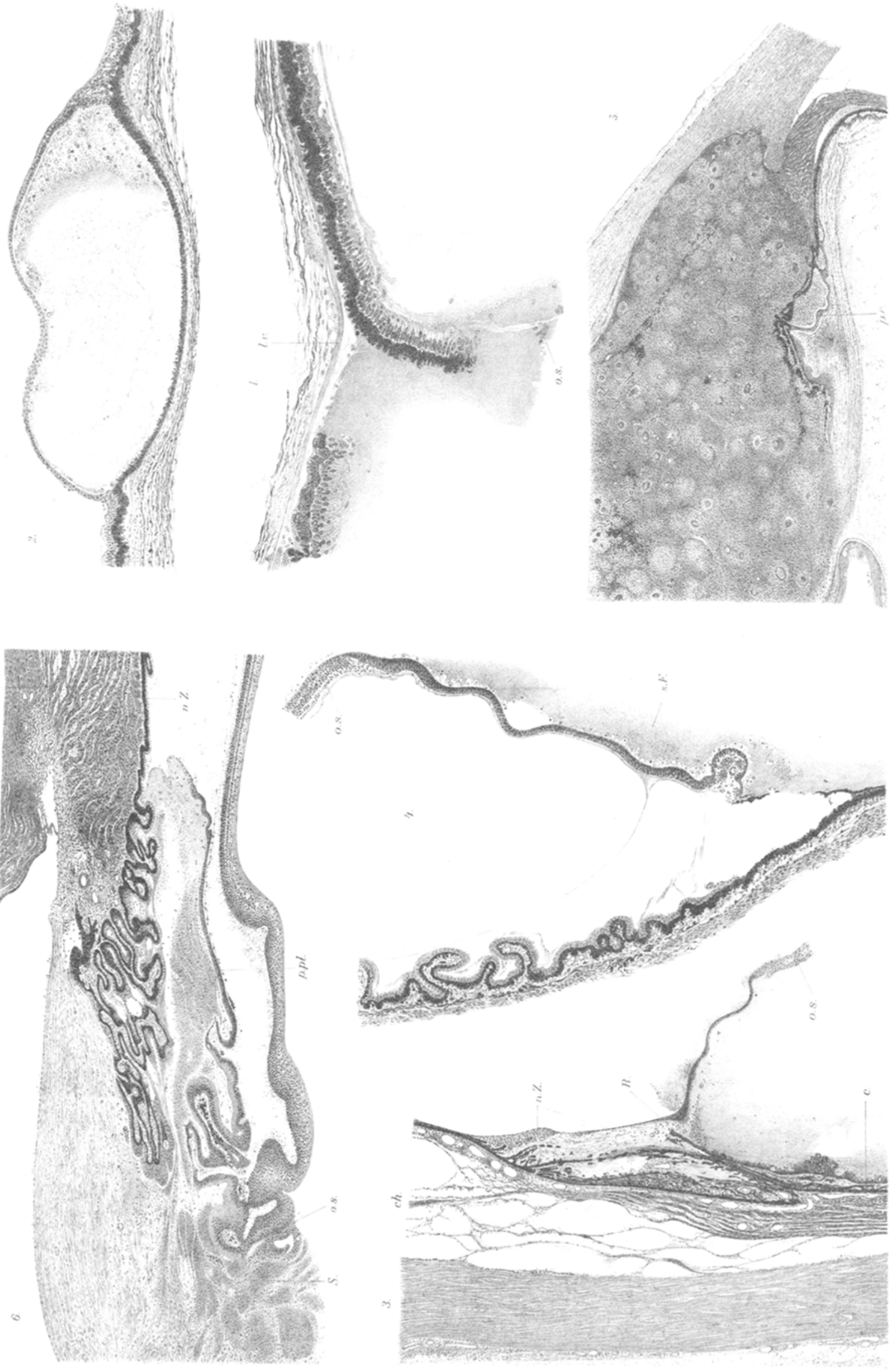